\title{
Poststroke Fatigue: Who Is at Risk for an Increase in Fatigue?
}

\author{
Hanna Maria van Eijsden, ${ }^{1}$ Ingrid Gerrie Lambert van de Port, ${ }^{1,2}$ \\ Johanna Maria August Visser-Meily, ${ }^{2}$ and Gert Kwakkel ${ }^{2,3}$ \\ ${ }^{1}$ Clinical Health Sciences, Faculty of Medicine, Utrecht University, 3508 TC, Utrecht, The Netherlands \\ ${ }^{2}$ Rudolf Magnus Institute of Neuroscience and Center of Excellence for Rehabilitation Medicine, University Medical Center Utrecht and \\ Rehabilitation Center De Hoogstraat, Rembrandtkade 10, 3582 TM Utrecht, The Netherlands \\ ${ }^{3}$ Department of Rehabilitation Medicine, Research Institute MOVE, VU University Medical Centre, \\ 1081 HV Amsterdam, The Netherlands
}

Correspondence should be addressed to Ingrid Gerrie Lambert van de Port, i.v.d.port@dehoogstraat.nl

Received 14 June 2011; Revised 22 July 2011; Accepted 15 August 2011

Academic Editor: Gillian Mead

Copyright ( 2012 Hanna Maria van Eijsden et al. This is an open access article distributed under the Creative Commons Attribution License, which permits unrestricted use, distribution, and reproduction in any medium, provided the original work is properly cited.

\begin{abstract}
Background. Several studies have examined determinants related to post-stroke fatigue. However, it is unclear which determinants can predict an increase in poststroke fatigue over time. Aim. This prospective cohort study aimed to identify determinants which predict an increase in post-stroke fatigue. Methods. A total of 250 patients with stroke were examined at inpatient rehabilitation discharge (T0) and 24 weeks later (T1). Fatigue was measured using the Fatigue Severity Scale (FSS). An increase in post-stroke fatigue was defined as an increase in the FSS score beyond the 95\% limits of the standard error of measurement of the FSS (i.e., 1.41 points) between $\mathrm{T} 0$ and $\mathrm{T} 1$. Candidate determinants included personal factors, stroke characteristics, physical, cognitive, and emotional functions, and activities and participation and were assessed at T0. Factors predicting an increase in fatigue were identified using forward multivariate logistic regression analysis. Results. The only independent predictor of an increase in poststroke fatigue was FSS (OR 0.50; 0.38-0.64, $P<0.001$ ). The model including FSS at baseline correctly predicted $7.9 \%$ of the patients who showed increased fatigue at T1. Conclusion. The prognostic model to predict an increase in fatigue after stroke has limited predictive value, but baseline fatigue is the most important independent predictor. Overall, fatigue levels remained stable over time.
\end{abstract}

\section{Introduction}

A common symptom after stroke is fatigue, with reported frequencies ranging from $38 \%$ to $77 \%$ [1], indicating that poststroke fatigue is a major problem after stroke. Forty percent of the patients considered fatigue to be one of the worst sequelae of stroke [2]. Patients feel unprepared for the fatigue phenomenon and struggle to adapt to it in daily life [3]. Fatigue has a debilitating influence on activities of daily living $[3,4]$ and is independently associated with health-related quality of life [4] and the resumption of paid work [5].

Several studies have examined determinants related to poststroke fatigue, but for many determinants there is inconclusive or insufficient evidence [1]. A recent prospective study demonstrated that baseline fatigue was the main predictor of the development of poststroke fatigue over time [6]. Conflicting evidence was found for personal factors such as gender [2, 7-12], age [2,6-12], and marital status $[7,8]$. A few studies found significant results for stroke characteristics, for example, previous stroke [7] and infratentorial infarctions $[6,11]$. A strong relationship between depression and post-stroke fatigue was described, both in crosssectional $[2,10,11]$ and longitudinal analyses [6-9]. However, poststroke fatigue can also occur in the absence of depression, and depression was also found independent of fatigue in stroke survivors $[2,10]$. A multivariate model including age, sex, locus of control, and depression explained $20 \%$ of the total variance of FSS scores 1 year after stroke [8]. This meant that the largest part of the variance in poststroke fatigue, $80 \%$, remained unexplained, suggesting that other determinants play a role in the occurrence of poststroke fatigue. One hypothesis is that physical deconditioning, which is common after stroke [13], might be associated 
with poststroke fatigue [14]. The influence of physical functioning has not been extensively investigated, and results have been inconsistent $[15,16]$.

Reported levels of poststroke fatigue are high and remain fairly stable over time within groups. At an individual level, however, significant changes do occur $[6,8]$. It might in fact be more relevant for clinical practice to identify those patients with a variable course of fatigue and especially the patients who are at risk for an increase in poststroke fatigue. Therefore, the aim of the present prospective cohort study was to identify determinants predicting increasing fatigue in patients after stroke. Candidate determinants include personal factors, stroke characteristics, physical, cognitive, and emotional functions, and activities and participation.

\section{Methods}

2.1. Design and Procedure. Data used in this study were collected between July 2008 and January 2011 as part of a large randomized controlled trial called FIT-Stroke (trial number NTR1534). The primary objective of the FIT-Stroke trial was to evaluate the effects on gait and the cost-effectiveness of a structured, progressive task-oriented circuit class training (CCT) program, compared to usual physical therapy care during outpatient rehabilitation in a rehabilitation center [17].

Patients were included in the study (T0) at the time of discharge from inpatient rehabilitation, when outpatient rehabilitation started. The followup assessment (T1) took place 24 weeks after discharge.

All measures were assessed by an independent researcher.

2.2. Participants. Inclusion criteria to participate in the study were (1) verified stroke according to the WHO definition [18]; (2) age $\geq 18$ years; (3) ability to walk a minimum of $10 \mathrm{~m}$ without physical assistance from a therapist (Functional Ambulation Categories $\geq 3$ ) [19]; (4) having been discharged home from a rehabilitation center; (5) giving informed consent. Patients were excluded if they (1) had a score on the Mini-Mental State Examination of less than 24 points [20]; (2) were unable to communicate (i.e., <4 points on the Utrechts Communicatie Onderzoek test) [21]; (3) lived more than $30 \mathrm{~km}$ from the rehabilitation center.

The study was approved by the Medical Ethics Committee of the University Medical Center Utrecht and all the participating rehabilitation centers. All included patients gave written informed consent.

\subsection{Measures}

2.3.1. Primary Outcome: Poststroke Fatigue. The impact of fatigue was measured by the Fatigue Severity Scale (FSS) [22]. The FSS consists of 9 items, with scores for each item ranging from 1 to 7 . The total FSS score is the mean of the 9 item scores [22]. Patients with a total score of $\geq 4$ points are classified as "fatigued" [23]. A reliability study with two independent observers and 18 stroke patients found an Intraclass Correlation Coefficient (ICC) for the FSS of 0.82 [4]. Item analysis showed excellent internal consistency and reliability for stroke patients (Cronbach's $\alpha=0.96$ ) [23]. In healthy subjects, the test-retest scores were stable over time [23]. The FSS scale was administered at T0 and T1. An increase in poststroke fatigue was defined as an increase in the FSS score beyond the 95\% limits of the standard error of measurement (SEM) of the FSS, SEM being defined as $\mathrm{SEM}=\mathrm{SD} * \sqrt{ } 1-\mathrm{ICC}$. The SD of the FSS was obtained from the current study. The ICC of the FSS used in our analysis was $0.82[4]$.

2.3.2. Candidate Determinants. Determinants were classified using the International Classification of Functioning, disability and health (ICF). All candidate determinants were assessed at T0.

2.3.3. Personal Factors. Data on age, sex, marital status, physical activity, and comorbidity before stroke were obtained at T0. A person was classified as "physically active before stroke" if he or she participated in moderate-intensity activity for at least 30 minutes a day, on five days a week [24]. Comorbidity was assessed by the Cumulative Illness Rating Scale (CIRS), which is a valid and reliable instrument that addresses all relevant body systems without using specific diagnoses [25]. The CIRS consists of 13 items, and the total score ranges from 0 (i.e., no morbidity) to 52 (very severe comorbidities).

2.3.4. Stroke Characteristics. Data on type of stroke, lateralization, time since stroke onset, and previous stroke were obtained from medical records at T0. Type of stroke was classified as ischemic versus hemorrhagic stroke. Lateralization was divided into three categories namely right hemisphere, left hemisphere, and other (e.g., brainstem, cerebellum).

2.3.5. Physical Functions. Strength was assessed by the Motricity Index (MI), which was used to determine the strength of the upper paretic limb (MI upper limb) and the lower paretic limb (MI lower limb). Scores range from 0 (no visual movement) to 100 (normal strength). The test has proven to be highly reliable and valid [26].

Strength was also assessed by the "strength" domain of the Stroke Impact Scale, version 3.0 (SIS). The SIS is a selfreported, stroke-specific measure that includes 59 items and assesses 8 domains relating to activities and participation [27]. SIS has shown excellent clinimetric properties in terms of concurrent and construct validity, test-retest reliability and responsiveness $[28,29]$. The SIS has been translated into Dutch, and the translated version also proved to be valid and responsive [30]. Subscale scores range from 0 to 100 percent [28].

Balance was tested by the Timed Balance Test (TBT). The TBT consists of 5 components scored on an ordinal scale and involves timed balance (i.e., 60 seconds) in five different positions of bilateral stance. One point is scored for each position maintained, so the score ranges from 0 to 5 . The test has been shown to be reliable and concurrent valid $[31,32]$.

2.3.6. Cognitive Functions. Cognition was assessed by the MMSE, a widely used brief screening instrument to 
determine orientation, memory, attention, calculation, language, and construction functions [20]. The score ranges from 0 to 30. A subject who scored less than 24 on the MMSE was considered to have cognitive impairments. Patients with an MMS $<24$ were excluded from the study.

Memory function was assessed by the "memory" domain of the SIS, with subscale scores range from 0 to 100 [28].

Inattention was measured by the Letter Cancellation Task and was regarded as positive when patients had two or more omissions on one side compared to the other side [33].

2.3.7. Emotional Functions. The Hospital Anxiety and Depression Scale (HADS) was used to determine mood, emotional distress, anxiety, depression, and emotional disorder. It is a brief, valid, reliable, and widely used instrument, known to produce meaningful results as a psychological screening tool. The HADS consists of 14 items (7 anxiety, 7 depression), each with a 4-point rating scale (0-3) and is responsive to change $[34,35]$. The depression and anxiety scales are analyzed as two separate domains, with scores for each scale ranging from 0 to 21.

Emotion was assessed by the corresponding domain on the SIS, with subscale scores range from 0 to 100 [28].

The Falls Efficacy Scale (FES) was used to measure fear of falling. The FES is based on the operational definition of this fear as "low perceived self-efficacy at avoiding falls during essential, nonhazardous activities of daily living" [36]. The score ranges from 0 to 130, with higher scores representing higher confidence and thus less anxiety.

2.3.8. Activities and Participation. Gait performance and endurance were assessed by the 6-Minute Walking Test (6 MWT), which has a good test-retest reliability (ICC = 0.973) [37-39].

The 5-Meter Timed Walking Test (5MTWT) was used to assess comfortable walking speed [40]. To reduce measurement error, we used the mean of three repeated walking speed measurements.

The Functional Ambulation Categories (FAC) instrument was used to assess walking ability. The scale includes six categories with scores ranging from 0 to 5 , that is, from unable to walk to independently walking without physical assistance $[19,41]$, though only patients with FAC 3 or higher were included in the trial.

Mobility was assessed by the Rivermead Mobility Index (RMI). The RMI consists of 14 questions and one observation (maximum score 15), covering aspects ranging from turning in bed to running [42]. Questions are simple and are scored dichotomously. The measure is reliable, valid, and responsive [42-44].

Extended activities of daily living (ADL) performance was assessed by the Nottingham Extended ADL (NEADL). The NEADL scale [45] is based on a self-reported questionnaire on levels of activity actually performed. The NEADL consists of 22 items in 4 domains (mobility, kitchen, domestic, and leisure). It has proven to be reliable and valid as an outcome measure in trials and observational studies. Each item is rated by one of four responses (able, able with difficulty, able with help, unable) and scores range from 0 to 66 .

Activity and participation domains of the SIS, that is, hand function, mobility, communication, ADL/IADL, and participation were also included in the analysis, with subscale scores ranging from 0 to 100 [28].

2.4. Statistics. Baseline characteristics were described using descriptive statistics (means and Standard Deviations (SDs); medians and ranges, odds ratio (95\% confidence interval)).

First, bivariate logistic regression analyses were conducted with the candidate determinants measured at T0. Candidate determinants with a significance level of $P<0.2$ were then selected for the forward multivariate logistic regression to identify independent predictors of an increase in fatigue at T1 (i.e., fatigue scores increasing beyond the $95 \%$ limits of the SEM). Multicollinearity was checked by means of Pearson correlation, with a correlation coefficient of $r>0.7$ being classified as multicollinearity. If the correlation coefficient was $>0.7$, the variable with the lowest coefficient, relative to the outcome measure was omitted. Goodness of fit of the multivariate logistic model was tested by the Hosmer Lemeshow test. A significance level of 0.05 was used to include a determinant in the model. We used a generally accepted rule of thumb for the maximum number of factors in a regression analysis, viz. one determinant was added to the equation for every 10 patients [46]. The present cohort participated in an intervention trial on the cost-effectiveness of circuit class training after stroke [17]. Preliminary results show that there are no time and interaction effects of treatment allocation with fatigue. The complete cohort was therefore included in the present analysis. Data were analyzed using SPSS for Windows version 16.0.

\section{Results}

3.1. Baseline Characteristics. Two hundred and fifty patients were included in the study, and 243 patients were still eligible at T1. Two patients died, two were excluded due to recurrent stroke, and three patients withdrew from the study. FSS scores at T1 were missing for one patient, so 242 patients were included in this analysis.

The mean age of the group as a whole $(N=242)$ was 57.1 years ( $\mathrm{SD}=10.3$ years); $64.9 \%$ of the patients were male. The average length of inpatient stay at the rehabilitation center was 72.1 days (SD 37.5). At the time of inclusion, the mean time since stroke onset was 97.0 days (SD 46.9).

3.2. Poststroke Fatigue. Fatigue was reported by $58.3 \%$ and $55.0 \%$ of the patients at T0 and T1, respectively. Mean FSS score was 4.1 (SD 1.7) at both measurements $(P=0.83)$. In $40.5 \%(N=98)$ of the patients, fatigue (FSS $\geq 4)$ was present at both measurements, while about a quarter $(N=66)$ of the patients reported no fatigue at either measurement. Over $50 \%$ of the patients reported that fatigue was one of the three most disabling symptoms after stroke (score $\geq 5$ on item 8 of the FSS). 
TABLE 1: Descriptive statistics for changes in fatigue over time $(N=$ 242).

\begin{tabular}{lccc}
\hline Condition & $N$ & $\begin{array}{c}\text { Mean FSS score } \\
\text { (SD) at T0 }\end{array}$ & $\begin{array}{r}\text { Mean FSS score } \\
\text { (SD) at T1 }\end{array}$ \\
\hline $\begin{array}{l}\text { Significant increase } \\
\text { in perception of } \\
\text { fatigue }\end{array}$ & 38 & $2.7(1.3)$ & $5.4(1.0)$ \\
$\begin{array}{l}\text { No significant } \\
\text { change in } \\
\text { perception of fatigue } \\
\begin{array}{l}\text { Significant decrease } \\
\text { in perception of } \\
\text { fatigue }\end{array}\end{array}$ & 43 & $5.2(1.1)$ & $4.3(1.6)$ \\
\hline
\end{tabular}

FSS: Fatigue Severity Scale, SD: Standard Deviation.

The $95 \%$ SEM of the FSS was $(1.7 * \sqrt{ }(1-0.82)) * 1.96=$ 1.41. A patient was classified as "having increased fatigue" when the score at T1 was 1.41 points higher than that at T0.

Using the $95 \%$ limits of the SEM, 38 patients $(15.7 \%)$ showed an increased fatigue score, while FSS scores had decreased in 43 patients $(17.8 \%)$, and the majority of the patients had remained stable in this respect $(66.5 \%)$. Table 1 shows FSS scores for each group at both baseline and followup.

3.3. Regression Analysis. Logistic regression analysis was conducted, comparing the group showing an increase in fatigue with the reference group (i.e., those with stable or decreased FSS scores). Baseline characteristics of both groups are reported in Table 2. Bivariate analysis of the determinants measured at T0 showed six determinants with $P<0.2$, viz. time since stroke, MI upper limb, 6MWT, MMSE, SIS memory, and FSS score at T0 (Table 2). Since there was no multicollinearity between these six determinants, all determinants were included in the multivariate logistic regression analysis. The only independent predictor of an increase in poststroke fatigue was the FSS score at T0 (OR 0.50 [0.380.64], Nagelkerke $\left.R^{2}=0.243\right)$. The multivariate model showed a good fit (Hosmer and Lemeshow test $P=0.495$ ). The model including FSS at baseline correctly predicted $7.9 \%$ (3 of 38) of the patients whose fatigue had increased at T1 and $96.6 \%$ (197 of 204) of the reference group.

\section{Discussion}

In our study, the FSS score measured at T0 was the only independent predictor of an increase in fatigue over time. Nevertheless, a model including the FSS score at T0 still poorly predicted an increase in poststroke fatigue. Our findings also indicate that poststroke fatigue is experienced by the majority of stroke patients, with 58.3 and $55.0 \%$ of our patients reporting fatigue at $\mathrm{T} 0$ and $\mathrm{T} 1$, respectively. A large proportion $(40.5 \%)$ of our patients were fatigued at both measurements, and $66.5 \%$ of the patients remained within the $95 \%$ limits of the SEM of the FSS, suggesting that the initial scores on the FSS are highly indicative for FSS scores at followup. This result further confirms that poststroke fatigue is a major problem after stroke, even in a relatively young and moderately affected population like ours, which remained fairly stable over time. However, the followup of the present study was restricted to 24 weeks. With that, it remains unclear if a longer followup would have resulted in the same conclusion.

A strong point of the current study was the large sample size of 250 participants and the use of a large variety of potential predictors at the different levels of the ICF. Some limitations need to be taken into account, however, when interpreting the results.

First, despite the large number of determinants included in the study, the multivariate regression model was a poor predictor of an increase in poststroke fatigue. This is in line with previously published studies $[8,12,15,16,47]$. In contrast to most other studies $[2,10,11,47,48]$, our study examined the physical determinants by means of physical performance tests instead of using nonperformance measures like the Oxford Handicap Scale, modified Rankin Scale, SF36, or Glasgow Outcome Scale. Our bivariate analysis suggested that the strength of the upper limb (MI) and the distance on the 6MWT were significantly related $(P<0.2)$ to an increase in fatigue over time. However, neither variable was included in the final multivariate model, suggesting that these physical determinants were not independent predictors of an increased fatigue score at $\mathrm{T} 1$.

The present study did not take determinants at the level of cognitive function and coping style sufficiently into account, which may have influenced the results. Previous studies found significant relations between poststroke fatigue and factors like cognition [48] and coping style [8, 48]. In our study, two of the three examined cognitive measures were included in the multivariate analysis, but they did not prove to be significantly related to an increase in poststroke fatigue. Although objective measures are generally preferred for cognition, we used a self-reported questionnaire and two global screening instruments, which may have influenced the results. Also, since we used the MMSE as an inclusion criterion, cognitive limitations in our sample were moderate.

Second, since there is no generally accepted definition of fatigue, there is no golden standard to measure poststroke fatigue either. Our study used the FSS to measure fatigue. Although this is a widely accepted and used scale to measure fatigue in stroke populations [1], this choice may have influenced the results.

Third, the use of an inception cohort at a fixed time after stroke onset is preferred in prognostic research, whereas our study took the baseline measurement at the time of discharge from inpatient rehabilitation, and there was a mean time interval between measurements of 96.9 days with a standard deviation of 46.9 days. Despite this, the frequency of poststroke fatigue found in our study is comparable to that reported in other studies using a similar timeframe $[1,8]$. Also, time since stroke onset turned out not to be an independent predictor of an increase in poststroke fatigue.

To our knowledge, this is the first study which specifically attempted to identify patients whose poststroke fatigue increased over time. The significant odds ratio of 0.5 found in our study suggests that patients with higher baseline FSS 
TABLE 2: Baseline characteristics and bivariate logistic regression analysis related to increased fatigue.

\begin{tabular}{|c|c|c|c|c|}
\hline & \multicolumn{2}{|c|}{ Baseline characteristics } & \multicolumn{2}{|c|}{ Bivariate logistic regression analysis } \\
\hline & $\begin{array}{c}\text { Deteriorated } \\
\quad N=38\end{array}$ & $\begin{array}{l}\text { Reference group* } \\
\qquad N=204\end{array}$ & OR $(95 \% \mathrm{CI})$ & $P$ value \\
\hline \multicolumn{5}{|l|}{ Personal factors } \\
\hline Age, mean \pm SD & $56.9 \pm 10.4$ & $57.1 \pm 10.3$ & $1.00(0.97-1.03)$ & 0.915 \\
\hline Male & $63.2 \%$ & $65.2 \%$ & $1.10(0.53-2.24)$ & 0.809 \\
\hline Physically active before Stroke (yes) & $81.6 \%$ & $78.9 \%$ & $1.88(0.63-5.63)$ & 0.261 \\
\hline CIRS, mean \pm SD & $6.1 \pm 3.7$ & $5.5 \pm 2.6$ & $1.07(0.95-1.21)$ & 0.251 \\
\hline Marital status; living with partner & $78.9 \%$ & $82.8 \%$ & $0.78(0.33-1.84)$ & 0.565 \\
\hline \multicolumn{5}{|l|}{ Stroke characteristics } \\
\hline Type of stroke; ischemic & $84.2 \%$ & $80.9 \%$ & $1.26(0.49-3.22)$ & 0.629 \\
\hline \multicolumn{5}{|l|}{ Lateralization } \\
\hline Right hemisphere (reference) & & & reference & reference \\
\hline Left hemisphere & $42.1 \%$ & $48.0 \%$ & $1.38(0.65-2.94)$ & 0.404 \\
\hline Other & & & $1.05(0.38-2.90)$ & 0.925 \\
\hline Time since stroke (days) & $108.8 \pm 53.9$ & $94.8 \pm 94.8$ & $1.06(0.999-1.01)$ & $0.094^{*}$ \\
\hline Previous stroke (yes) & $10.5 \%$ & $10.8 \%$ & $0.812(0.43-1.51)$ & 0.513 \\
\hline \multicolumn{5}{|l|}{ Physical functions } \\
\hline MI upper limb, mean \pm SD & $55.1 \pm 29.3$ & $61.3 \pm 25.8$ & $0.99(0.98-1.00)$ & $0.185^{*}$ \\
\hline MI lower limb, mean \pm SD & $67.4 \pm 21.4$ & $68.1 \pm 20.1$ & $1.00(0.98-1.02)$ & 0.843 \\
\hline SIS-strength, mean $\pm \mathrm{SD}$ & $53.6 \pm 21.3$ & $51.5 \pm 19.9$ & $1.01(0.99-1.02)$ & 0.546 \\
\hline TBT, median (range) & $3.5(1-5)$ & $3(0-5)$ & $0.89(0.65-1.23)$ & 0.491 \\
\hline \multicolumn{5}{|l|}{ Cognitive functions } \\
\hline MMSE, mean \pm SD & $28.5 \pm 1.6$ & $28.0 \pm 1.7$ & $1.21(0.96-1.51)$ & $0.104^{*}$ \\
\hline SIS-memory, mean \pm SD & $85.3 \pm 13.9$ & $81.4 \pm 17.8$ & $1.02(0.99-1.04)$ & $0.194^{*}$ \\
\hline Inattention (yes) & $13.2 \%$ & $21.6 \%$ & $0.55(0.20-1.50)$ & 0.242 \\
\hline \multicolumn{5}{|l|}{ Psychological characteristics } \\
\hline HADS-depression, mean \pm SD & $4.6 \pm 3.6$ & $4.8 \pm 3.4$ & $0.98(0.88-1.09)$ & 0.706 \\
\hline HADS-anxiety, mean \pm SD & $3.3 \pm 2.8$ & $3.8 \pm 3.5$ & $0.95(0.85-1.06)$ & 0.338 \\
\hline SIS-emotion, mean \pm SD & $80.6 \pm 13.4$ & $82.8 \pm 13.7$ & $1.00(0.97-1.01)$ & 0.353 \\
\hline FES, mean \pm SD & $97.1 \pm 21.0$ & $97.3 \pm 19.2$ & $1.00(0.98-1.02)$ & 0.955 \\
\hline FSS, mean \pm SD & $2.7 \pm 1.3$ & $4.4 \pm 1.6$ & $0.50(0.38-0.64)$ & $0.000^{*}$ \\
\hline \multicolumn{5}{|l|}{ Activities and participation } \\
\hline $6 \mathrm{MWT}$, mean distance $\pm \mathrm{SD}$ & $279.1 \pm 132.1$ & $326.6 \pm 126.0$ & $1.00(0.99-1.00)$ & $0.037^{*}$ \\
\hline $5 \mathrm{MTWT}$, mean time $\pm \mathrm{SD}$ & $9.0 \pm 6.1$ & $8.1 \pm 7.9$ & $1.01(0.97-1.05)$ & 0.521 \\
\hline FAC, median (range) & $5(4-5)$ & $5(3-5)$ & $0.78(0.45-1.34)$ & 0.361 \\
\hline RMI, mean $\pm \mathrm{SD}$ & $12.4 \pm 1.5$ & $12.5 \pm 1.9$ & $0.99(0.82-1.20)$ & 0.930 \\
\hline NEADL, mean \pm SD & $32.3 \pm 10.5$ & $34.0 \pm 11.1$ & $0.99(0.96-1.02)$ & 0.372 \\
\hline SIS-mobility, mean \pm SD & $79.0 \pm 14.9$ & $79.2 \pm 14.2$ & $1.00(0.98-1.02)$ & 0.940 \\
\hline SIS-hand function, mean \pm SD & $41.4 \pm 38.7$ & $45.5 \pm 34.8$ & $1.00(0.99-1.01)$ & 0.520 \\
\hline SIS-ADL/IADL, mean \pm SD & $67.1 \pm 16.0$ & $70.4 \pm 15.2$ & $0.99(0.97-1.01)$ & 0.238 \\
\hline SIS-communication, mean \pm SD & $83.4 \pm 22.8$ & $85.2 \pm 18.2$ & $1.00(0.79-1.01)$ & 0.579 \\
\hline SIS-participation, mean \pm SD & $63.4 \pm 24.3$ & $67.0 \pm 20.3$ & $0.99(0.98-1.01)$ & 0.329 \\
\hline
\end{tabular}

${ }^{*}$ Reference group: those with stable or decreased FSS scores; CIRS: Cumulative Illness Rating Scale, MI: Motricity Index, SIS: Stroke Impact Scale, TBT: Timed Balance Test, MMSE: Mini-Mental State Examination, HADS: Hospital Anxiety Depression Scale, FES: Falls Efficacy Scale, FSS: Fatigue Severity Scale, 6MWT: 6-Minute Walking Test, 5MTWT: 5-Meter Timed Walking Test, FAC: Functional Ambulation Categories, RMI: Rivermead Mobility Index, NEADL: Nottingham extended activities of daily living, CI: Confidence Interval ${ }^{*} P$ value $<0.2$, included in the multivariate logistic regression analysis. 
scores are less likely to show increased fatigue (i.e., having a higher FSS score) in the long term. This is in contrast to the findings by Snaphaan et al. who reported that a higher fatigue score at baseline was related to a change from no fatigue at baseline to the presence of fatigue at followup (incident fatigue) [6]. Although the biological explanation for our finding remains unclear, the decreased likelihood to show an increase in FSS beyond the 95\% limits of the SEM may be caused by a ceiling effect of the FSS and hence regression to the mean. Patients with high initial scores on a scale are more likely to show declining scores, whereas patients with very low scores at baseline are more likely to show an increase at a second assessment.

A significant relation between poststroke fatigue and depression has been shown in several studies $[2,7,8,10]$. The study by Snaphaan et al. suggested that patients with fatigue at followup but not at baseline had higher baseline scores for depression compared to patients with no fatigue at either of these times [6]. The strong relation between fatigue and depression is consistent with the fact that fatigue is a symptom of depression. However, it has been shown that fatigue can occur without the presence of depression [8]. In our study, no significant relationship was found between depression and an increase in poststroke fatigue over time.

An ability to identify risk factors for an increase in poststroke fatigue will benefit efforts to design treatment modalities and counsel patients and their relatives. Currently, there is insufficient evidence to decide which treatment, whether pharmaceutical or by (multidisciplinary) rehabilitation, would be preferable [49]. Further research, for example, welldesigned randomized controlled trials, will be necessary to show which interventions can be effective. Recently published preliminary results of a randomized controlled trial on the effect of combined graded physical activity training and cognitive treatment to treat poststroke fatigue show a significant decline in fatigue severity immediately after the treatment as well as 6 months after treatment [50]. Since coping style is an important factor in poststroke fatigue $[8,48]$, this is an important aspect to consider for inclusion in treatment. In addition to further exploration of effective treatment modalities, further research is needed to examine the determinants related to poststroke fatigue. The role of physical functioning should be further explored, since this has hardly been included in prognostic research so far. Poststroke fatigue is a multifactorial phenomenon which is probably not captured by one single outcome measurement. Therefore, future studies should consider including determinants of different domains and using various outcome measurements to determine the different dimensions of fatigue.

\section{Conclusion}

Baseline fatigue is the only independent predictor of an increase in poststroke fatigue, and predicting poststroke fatigue remains difficult. Most patients remain stable over time, meaning that the initial FSS scores are indicative of followup scores. A high percentage of our relatively young and moderately affected sample suffered from poststroke fatigue, indicating that this is a major problem in this group of stroke survivors.

\section{References}

[1] A. Lerdal, L. N. Bakken, S. E. Kouwenhoven et al., "Poststroke fatigue-a review," Journal of Pain and Symptom Management, vol. 38, no. 6, pp. 928-949, 2009.

[2] J. L. Ingles, G. A. Eskes, and S. J. Phillips, "Fatigue after stroke," Archives of Physical Medicine and Rehabilitation, vol. 80, no. 2, pp. 173-178, 1999.

[3] N. A. Flinn and J. E. Stube, "Post-stroke fatigue: qualitative study of three focus groups," Occupational Therapy International, vol. 17, no. 2, pp. 81-91, 2010.

[4] I. G. L. Van De Port, G. Kwakkel, V. P. M. Schepers, C. T. I. Heinemans, and E. Lindeman, "Is fatigue an independent factor associated with activities of daily living, instrumental activities of daily living and health-related quality of life in chronic stroke?" Cerebrovascular Diseases, vol. 23, no. 1, pp. 40-45, 2007.

[5] G. Andersen, D. Christensen, M. Kirkevold, and S. P. Johnsen, "Post-stroke fatigue and return to work: a 2-year follow-up," Acta Neurologica Scandinavica. In press.

[6] L. Snaphaan, S. van der Werf, and F.-E. de Leeuw, "Time course and risk factors of post-stroke fatigue: a prospective cohort study," European Journal of Neurology, vol. 18, no. 4, pp. 611-617, 2011.

[7] E. L. Glader, B. Stegmayr, and K. Asplund, "Poststroke fatigue: a 2-year follow-up study of stroke patients in Sweden," Stroke, vol. 33, no. 5, pp. 1327-1333, 2002.

[8] V. P. Schepers, A. M. Visser-Meily, M. Ketelaar, and E. Lindeman, "Poststroke fatigue: course and its relation to personal and stroke-related factors," Archives of Physical Medicine and Rehabilitation, vol. 87, no. 2, pp. 184-188, 2006.

[9] P. Appelros, "Prevalence and predictors of pain and fatigue after stroke: a population-based study," International Journal of Rehabilitation Research, vol. 29, no. 4, pp. 329-333, 2006.

[10] S. Choi-Kwon, S. W. Han, S. U. Kwon, and J. S. Kim, "Poststroke fatigue: characteristics and related factors," Cerebrovascular Diseases, vol. 19, no. 2, pp. 84-90, 2005.

[11] H. Naess, H. I. Nyland, L. Thomassen, J. Aarseth, and K. M. Myhr, "Fatigue at long-term follow-up in young adults with cerebral infarction," Cerebrovascular Diseases, vol. 20, no. 4, pp. 245-250, 2005.

[12] G. E. Mead, C. Graham, P. Dorman et al., "Fatigue after stroke: baseline predictors and influence on survival. analysis of data from UK patients recruited in the international stroke trial," PLoS One, vol. 6, no. 3, Article ID e16988, 2011.

[13] F. M. Ivey, R. F. Macko, A. S. Ryan, and C. E. Hafer-Macko, "Cardiovascular health and fitness after stroke," Topics in Stroke Rehabilitation, vol. 12, no. 1, pp. 1-16, 2005.

[14] D. Christensen, S. P. Johnsen, T. Watt, I. Harder, M. Kirkevold, and G. Andersen, "Dimensions of post-stroke fatigue: a twoyear follow-up study," Cerebrovascular Diseases, vol. 26, no. 2, pp. 134-141, 2008.

[15] S. J. Lewis, A. J. Barugh, C. A. Greig et al., "Is fatigue after stroke associated with physical deconditioning? A cross-sectional study in ambulatory stroke survivors," Archives of Physical Medicine and Rehabilitation, vol. 92, no. 2, pp. 295-298, 2011.

[16] K. M. Michael, J. K. Allen, and R. F. Macko, "Fatigue after stroke: relationship to mobility, fitness, ambulatory activity, social support, and falls efficacy," Rehabilitation Nursing, vol. 31, no. 5, pp. 210-217, 2006. 
[17] I. G. van de Port, L. Wevers, H. Roelse, L. van Kats, E. Lindeman, and G. Kwakkel, "Cost-effectiveness of a structured progressive task-oriented circuit class training programme to enhance walking competency after stroke: the protocol of the FIT-Stroke trial," BMC Neurology, vol. 9, article 43, 2009.

[18] Stroke, "Recommendations on stroke prevention, diagnosis, and therapy. Report of the WHO Task Force on Stroke and other Cerebrovascular Disorders," Stroke, vol. 20, no. 10, pp. 1407-1431, 1989.

[19] M. K. Holden, K. M. Gill, and M. R. Magliozzi, "Clinical gait assessment in the neurologically impaired. Reliability and meaningfulness," Physical Therapy, vol. 64, no. 1, pp. 35-40, 1984.

[20] M. F. Folstein, S. E. Folstein, and P. R. McHugh, “'Mini mental state. A practical method for grading the cognitive state of patients for the clinician," Journal of Psychiatric Research, vol. 12, no. 3, pp. 189-198, 1975.

[21] E. M. Pijfers, L. A. De Vries, and H. Messing-Petersen, Het Utrechts Communicatie Onderzoek, 1985.

[22] L. B. Krupp, N. G. LaRocca, J. Muir-Nash, and A. D. Steinberg, "The fatigue severity scale. Application to patients with multiple sclerosis and systemic lupus erythematosus," Archives of Neurology, vol. 46, no. 10, pp. 1121-1123, 1989.

[23] P. O. Valko, C. L. Bassetti, K. E. Bloch, U. Held, and C. R. Baumann, "Validation of the fatigue severity scale in a Swiss cohort," Sleep, vol. 31, no. 11, pp. 1601-1607, 2008.

[24] H. Kemper, W. Ooijendijk, and M. Stiggelbout, "Consensus over de Nederlandse norm voor gezond bewegen," Tijdschrift voor Sociale Gezondheidszorg, vol. 78, no. 3, pp. 180-183, 2000.

[25] V. De Groot, H. Beckerman, G. J. Lankhorst, and L. M. Bouter, "How to measure comorbidity: a critical review of available methods," Journal of Clinical Epidemiology, vol. 56, no. 3, pp. 221-229, 2003.

[26] C. Collin and D. Wade, "Assessing motor impairment after stroke: a pilot reliability study," Journal of Neurology Neurosurgery and Psychiatry, vol. 53, no. 7, pp. 576-579, 1990.

[27] P. W. Duncan, R. K. Bode, S. M. Lai, and S. Perera, "Rasch analysis of a new stroke-specific outcome scale: the stroke impact scale," Archives of Physical Medicine and Rehabilitation, vol. 84, no. 7, pp. 950-963, 2003.

[28] P. W. Duncan, D. Wallace, S. M. Lai, D. Johnson, S. Embretson, and L. J. Laster, "The stroke impact scale version 2.0: evaluation of reliability, validity, and sensitivity to change," Stroke, vol. 30, no. 10, pp. 2131-2140, 1999.

[29] P. W. Duncan, S. M. Lai, D. Tyler, S. Perera, D. M. Reker, and S. Studenski, "Evaluation of proxy responses to the Stroke Impact Scale," Stroke, vol. 33, no. 11, pp. 2593-2599, 2002.

[30] I. van de Port, K. Leenes, D. Sellmeijer, A. Zuidgeest, and G. Kwakkel, "Betrouwbaarheid en concurrente validiteit van de Nederlandse Stroke Impact Scale 2.0 bij patiënten met een CVA," Nederlands Tijdschrift voor Fysiotherapie, vol. 118, no. 1, pp. 12-18, 2008.

[31] R. W. Bohannon, P. A. Larkin, and A. C. Cook, "Decrease in timed balance test scores in aging," Physical Therapy, vol. 64, no. 7, pp. 1067-1070, 1984.

[32] R. W. Bohannon, S. Walsh, and M. C. Joseph, "Ordinal and timed balance measurements: reliability and validity in patients with stroke," Clinical Rehabilitation, vol. 7, no. 1, pp. 9-14, 1993.

[33] M. D. Lezak and M. D. Lezak, Neuropsychological Assessment, Oxford University Press, Oxford, UK, 2004.

[34] A. S. Zigmond and R. P. Snaith, "The hospital anxiety and depression scale," Acta Psychiatrica Scandinavica, vol. 67, no. 6, pp. 361-370, 1983.
[35] C. Herrmann, "International experiences with the hospital anxiety and depression scale - a review of validation data and clinical results," Journal of Psychosomatic Research, vol. 42, no. 1, pp. 17-41, 1997.

[36] M. E. Tinetti, D. Richman, and L. Powell, "Falls efficacy as a measure of fear of falling," Journals of Gerontology, vol. 45, no. 6, pp. P239-P243, 1990.

[37] S. Tyson and L. Connell, "The psychometric properties and clinical utility of measures of walking and mobility in neurological conditions: a systematic review," Clinical Rehabilitation, vol. 23, no. 11, pp. 1018-1033, 2009.

[38] G. D. Fulk, J. L. Echternach, L. Nof, and S. O’Sullivan, “Clinometric properties of the six-minute walk test in individuals undergoing rehabilitation poststroke," Physiotherapy Theory and Practice, vol. 24, no. 3, pp. 195-204, 2008.

[39] G. H. Guyatt, M. J. Sullivan, and P. J. Thompson, “The 6-minute walk: a new measure of exercise capacity in patients with chronic heart failure," Canadian Medical Association Journal, vol. 132, no. 8, pp. 919-921, 1985.

[40] N. M. Salbach, N. E. Mayo, J. Higgins, S. Ahmed, L. E. Finch, and C. L. Richards, "Responsiveness and predictability of gait speed and other disability measures in acute stroke," Archives of Physical Medicine and Rehabilitation, vol. 82, no. 9, pp. 1204-1212, 2001.

[41] B. Kollen, G. Kwakkel, and E. Lindeman, "Time dependency of walking classification in stroke," Physical Therapy, vol. 86, no. 5, pp. 618-625, 2006.

[42] F. M. Collen, D. T. Wade, G. F. Robb, and C. M. Bradshaw, "The Rivermead mobility index: a further development of the Rivermead motor assessment," International Disability Studies, vol. 13, no. 2, pp. 50-54, 1991.

[43] C. L. Hsieh, I. P. Hsueh, and H. F. Mao, "Validity and responsiveness of the Rivermead Mobility Index in stroke patients," Scandinavian Journal of Rehabilitation Medicine, vol. 32, no. 3 , pp. 140-142, 2000.

[44] I. P. Hsueh, C. H. Wang, C. F. Sheu, and C. L. Hsieh, "Comparison of psychometric properties of three mobility measures for patients with stroke," Stroke, vol. 34, no. 7, pp. 1741-1745, 2003.

[45] F. M. Nouri and N. B. Lincoln, "An extended activities of daily living scale for stroke patients," Clinical Rehabilitation, vol. 1, pp. 301-305, 1987.

[46] P. Peduzzi, J. Concato, E. Kemper, T. R. Holford, and A. R. Feinstem, "A simulation study of the number of events per variable in logistic regression analysis," Journal of Clinical Epidemiology, vol. 49, no. 12, pp. 1373-1379, 1996.

[47] A. Lerdal, L. N. Bakken, E. F. Rasmussen et al., "Physical impairment, depressive symptoms and pre-stroke fatigue are related to fatigue in the acute phase after stroke," Disability and Rehabilitation, vol. 33, no. 4, pp. 334-342, 2011.

[48] P. E. C. A. Passier, M. W. M. Post, M. J. E. van Zandvoort, G. J. E. Rinkel, E. Lindeman, and J. M. A. Visser-Meily, "Predicting fatigue 1 year after aneurysmal subarachnoid hemorrhage," Journal of Neurology, pp. 1-7, 2011.

[49] E. McGeough, A. Pollock, L. N. Smith et al., "Interventions for post-stroke fatigue," Cochrane Database of Systematic Reviews, no. 3, Article ID CD007030, 2009.

[50] A. M. E. E. Zedlitz, L. Fasotti, and A. C. H. Geurts, "Post-stroke fatigue: a treatment protocol that is being evaluated," Clinical Rehabilitation, vol. 25, no. 6, pp. 487-500, 2011. 


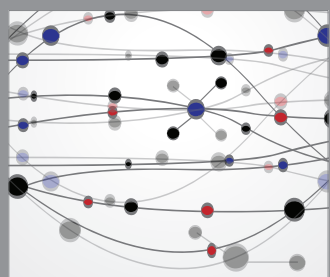

The Scientific World Journal
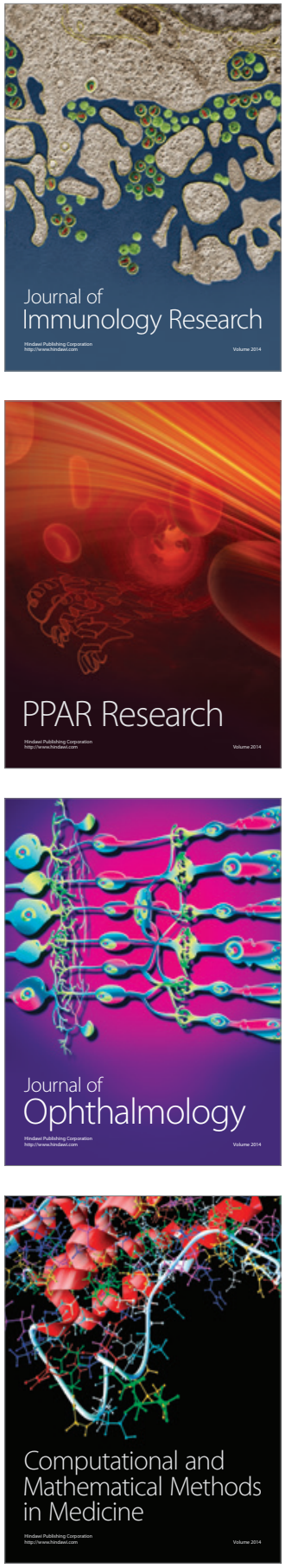

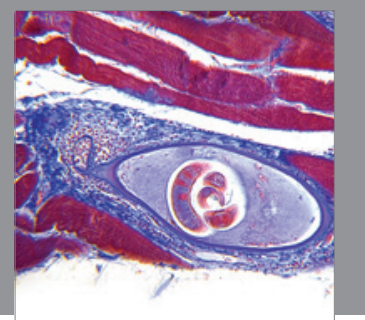

Gastroenterology

Research and Practice
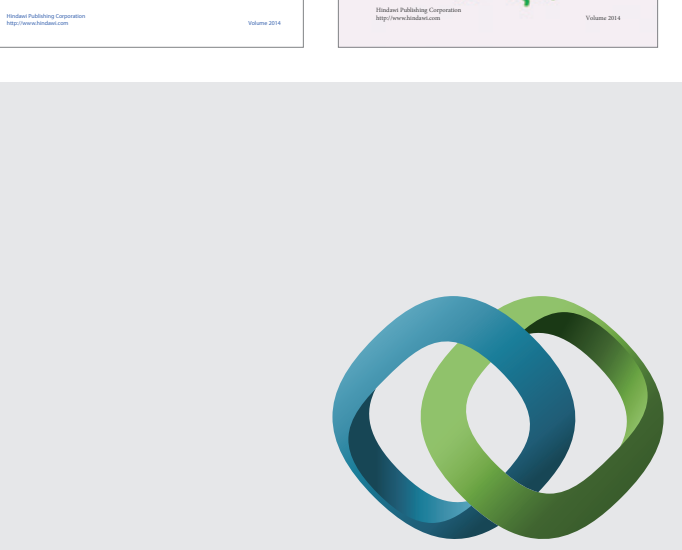

\section{Hindawi}

Submit your manuscripts at

http://www.hindawi.com
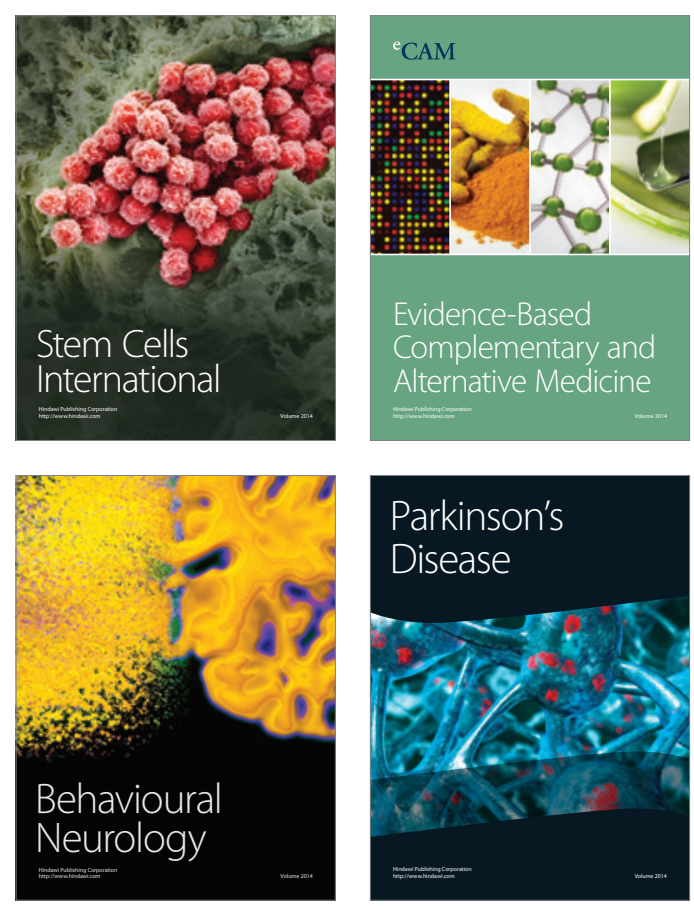

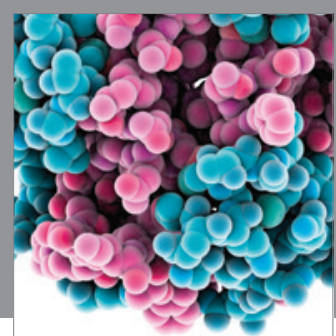

Journal of
Diabetes Research

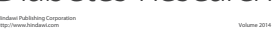

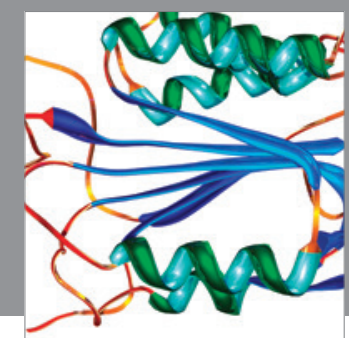

Disease Markers
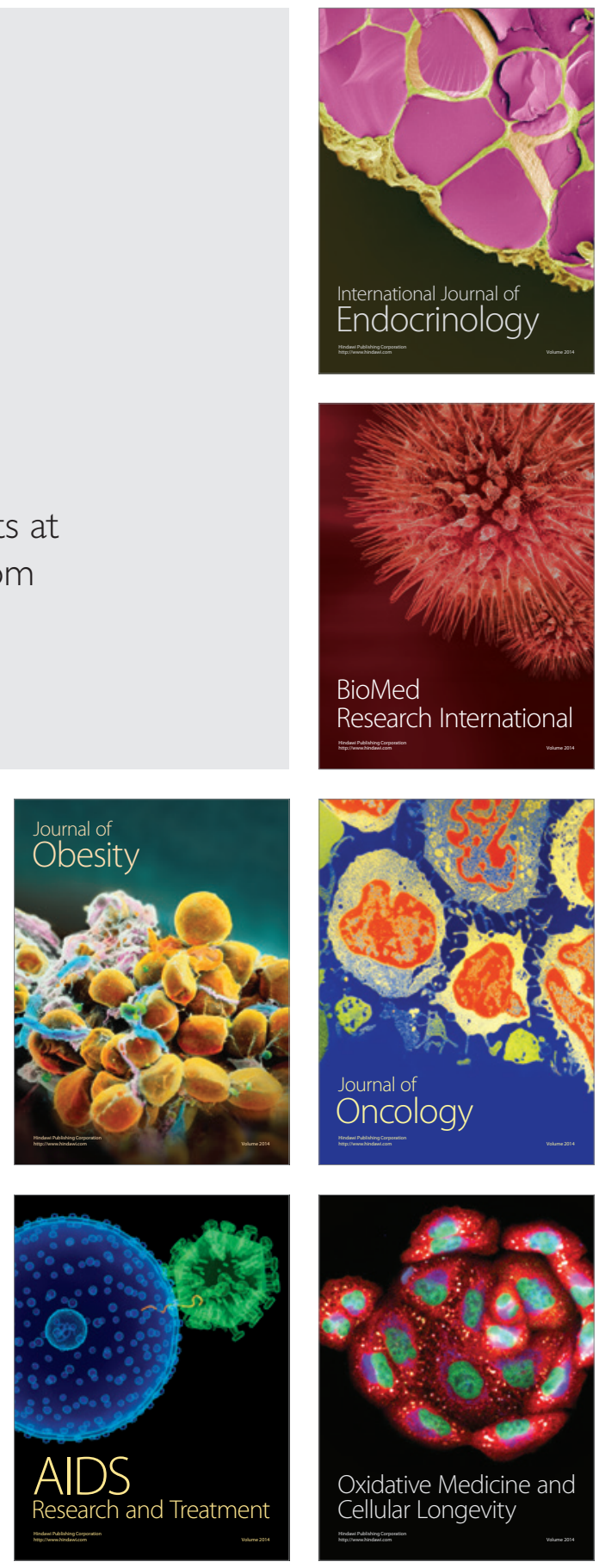